\title{
Identification, Characterization, and Hydrolase Producing Performance of Thermophilic Bacteria: Geothermal Hot Springs in Eastern and Southeastern Anatolia Region of Turkey
}

Orhan Uluçay ( $\nabla$ orhanulucay@gmail.com )

Kafkas University: Kafkas Universitesi https://orcid.org/0000-0002-0820-5372

Arzu Görmez

Erzurum Technical University

Cem Öziç

Kafkas University

\section{Research Article}

Keywords: Bacillus subtilis, Enzyme activity, Geobacillus, Hot springs, Thermophilic bacteria

Posted Date: April 16th, 2021

DOI: https://doi.org/10.21203/rs.3.rs-348608/v1

License: @ (i) This work is licensed under a Creative Commons Attribution 4.0 International License. Read Full License

Version of Record: A version of this preprint was published at Antonie van Leeuwenhoek on January 15th, 2022. See the published version at https://doi.org/10.1007/s10482-021-01678-5. 


\section{Abstract}

In the last twenty years, researchers have increasingly focused on the rich microorganism-based diversity of natural hot spring resources to explore the benefits of thermophiles in industrial and biotechnological fields. For this purpose, a total of 83 thermophilic bacilli were isolated from 7 different geothermal hot springs (at temperatures ranging between 40 and $85 \circ$ C) located in the east and Southeastern of Turkey. These isolates were identified by different methods such as physiological, morphological, biochemical, molecular properties. According to 16S rRNA gene sequence analysis, 5 different isolates ( Bacillus coagulans, Bacillus licheniformis, Bacillus subtilis, Bacillus thuringiensis, and Geobacillus kaustophilus ) were identified. It was observed that B. licheniformis and B. subtilis were the most species obtained from the researched hot spring sources. Phylogenetic relationships of isolates were evaluated with the help of a phylogenetic tree. The conditions of bacterial isolates to synthesize various hydrolytic enzymes such as protease, cellulase, lipase, and amylase were investigated. When the potential of isolates to produce hydrolytic enzymes was examined, protease 73 ( $88 \%$ ), cellulase 34 (41\%), lipase 69 (83\%), and amylase 68 (82\%) were detected. All isolates have at least one or more extracellular protease, cellulase, amylase, or lipase activity. Besides, $32.8 \%$ (27) of bacterial isolates were able to synthesize all of the hydrolytic enzymes.

\section{Introduction}

Geothermal hot springs generally host microorganisms that can be active and produce various enzymes under extreme conditions, depending on their environment (Mehta et al. 2016, Panosyan et al. 2020, Short 1998). The microorganisms and their enzymes, products which are the main target of alternative sources, are often used in many industrial areas. These enzymes in the biotech industry; it is widely used in many fields such as food processing, medicine, molecular biology, vaccine development, genetics, renewable energy, organic synthesis, and biofuel production (Eriksen 2008, Khan and Sathya 2018, Zakzeski et al. 2010).

Thermophilic microorganisms can thrive in different ecological habitats, both aquatic and terrestrial. These habitats are known as hydrothermal facilities, sugar heaps, hot springs, deep seas, and volcanic regions. Hot springs are one of the most important habitats for thermophilic microorganisms (Aanniz et al. 2015). Therefore, thermophilic microorganisms attract the attention of researchers as an important source of new and high-temperature resistant enzymes such as xylanases, DNA polymerases, amylases, cellulases, phytases, chitinases, proteases, lipases, and pectinases (Falcicchio et al. 2021, Lee et al. 2010, Luo et al. 2017, Msarah et al. 2020, Tango and Islam 2002). Although the necessary equipment and approaches have changed in parallel with the development of molecular biology in the determination of microorganisms that produce these enzymes in recent years, it is still important to perform physiological, morphological, and biochemical analyzes for pure culture isolation studies (Abdollahi et al. 2021, Akmar et al. 2011, Bouacem et al. 2021, Chan et al. 2015, Ifandi and Alwi 2018, Kılınç et al. 2021, Tirawongsaroj et al. 2008). In many studies, it was demonstrated that thermophilic microorganisms shave different phylogenetic varieties which include both bacteria and representatives of the archaea domain. In the bacteria domain, it is known that the most isolated thermophilic strains belonged to Bacillus genus (Abootalebi et al. 2020, Ladeira et al. 2015, Shen et al. 2021, Verma et al. 2018, Wang et al. 2006). The presence of thermophilic bacilli has been investigated in many different hot springs around the world at different times including Turkey (Yanmis et al. 2015) India (Verma et al. 2018), Iran (Abootalebi et al. 2020), Indonesia (Safitri et al. 2020), Jordanian (Mohammad et al. 2017), Faisalabad (Saleh et al. 2020), Tunisia (Salem et al. 2020), Brazil (Bernardo et al. 2020), Egypt (Saeed et al. 2020), Malaysian (Msarah et al. 2020), Vietnam (Dang et al. 2018), Saudi Arabia (Al-Johani et al. 2016), Japan (Akita et al. 2017) and other countries.

Turkey is one of the world's most important countries because of its many thermal water reserves (Oztas Gulmus and Gormez 2020b). Hot springs in the east and southeast of Turkey are the reservoir of natural resources for biotechnological based study (Guven et al. 2018). In many studies, different bacteria and archaea have been reported analysis of microorganisms got from thermal source east and southeast of Turkey Hasanabdal (Poli et al. 2012, Savas et al. 2009, Yanmis et al. 2015), Sirnak (Derya and Ahmet 2014), Diyadin (Baltaci et al. 2017), Dargecit (Acer et al. 2015), Guclukonak (Poli et al. 2012), and Pasinler (Adiguzel et al. 2011, Oztas Gulmus and Gormez 2020b). It is also observed that many of the isolates belong to the thermophilic Bacillus and Geobacillus (Oztas Gulmus and Gormez 2020b, Yilmaz et al. 2016). Most of the enzymes produced by these microorganisms in extreme conditions and which are especially important in the hydrolysis of biomass have not been discovered, yet (Thakur et al. 2021). Therefore, it is necessary to isolate and investigate new bacilli in the production of biotechnologically thermostable enzymes (Margaryan et al. 2018).

In this study, it was aimed to isolate bacteria from 7 hot springs at high altitude in Eastern and Southeastern Anatolia regions, to identify them by conventional and molecular methods, to determine the optimum growth conditions of the isolates, and to analyze for their capability to produce hydrolase enzymes such as protease, amylase, lipase, and cellulase.

\section{Materials And Methods}

\section{Collection of biological material and isolation of bacteria}

The soil/sediment, mud, and water samples were collected from 7 hot springs in the Eastern and Southeastern provinces of Turkey including Agri (Diyadin, Davut, and Kopru; Lat.: 39,493548, Lng.: 43,649362), Erzurum (Pasinler; Lat.: 39,978194 Lng.: 41,664286), Van (Hasanabdal Lat.: 39,225049, Lng.: 43,388236), Siirt (Hista Lat.: 37,707582, Lng.: 42,002020, Mardin (Dargecit Lat.: 37,546184, Lng.: 41,720381) and Sirnak (Guclukonak Lat.: 37,476818, Lng.: 41,939351) in August and September 2016. The locations of the different geothermal resources in this study are shown in the map in Fig. 1. The samples were collected from the shallow and muddy areas at the outlet of the hot springs in sterile cap bottles surrounded by an aluminum foil in a way not to receive sunlight. The temperature and $\mathrm{pH}$ parameters of the spring waters were determined by a portable device that can measure both $\mathrm{pH}$ and temperature (AZ 8685 ). The collected samples from each location were transferred to sterile tubes containing physiological saline water $(0.85 \mathrm{~g} \mathrm{NaCl}$ and $100 \mathrm{~mL}$ distilled water). $0.1 \mathrm{~mL}$ of diluent from each Eppendorf was transferred to Luria Bertani (LB) (Sigma-Aldrich) culture medium and $100 \mathrm{~mL}$ Erlenmeyer flasks and incubated at different temperatures $\left(40^{\circ} \mathrm{C}, 55^{\circ} \mathrm{C}, 65^{\circ} \mathrm{C}, 70^{\circ} \mathrm{C}, 75^{\circ} \mathrm{C}, 85^{\circ} \mathrm{C}\right.$, and $90^{\circ} \mathrm{C}$ ) for $24-72$ hours. Single colonies forming different colony morphologies were determined, and 
these pure isolates were streaked again on solid media. The purified isolates were preserved in an LB medium containing $15 \%$ glycerol at $-80{ }^{\circ} \mathrm{C}$ until use in subsequent studies (Gormez et al. 2013).

\section{Conventional characterization of bacteria}

The colony morphology and motility tests of all isolates were examined by light microscope (Best Scopes BS-2030t + BLC - 450). Morphological properties of isolated bacteria were determined by Gram staining, endospore formation, and mobility tests using standard procedures (Bernard et al. 2017). Physiological properties (temperature, $\mathrm{pH}$, and $\mathrm{NaCl}$ concentrations) of all isolates were tested at various stages of growth under aerobic conditions (Oztas Gulmus and Gormez 2020b). For this purpose, all the isolates were incubated at different temperatures $\left(40-90{ }^{\circ} \mathrm{C}\right)$, different $\mathrm{pH}$ levels $(4.0-12.0)$ and variable $\mathrm{NaCl}$ concentrations (up to $10 \% \mathrm{w} / \mathrm{v}$ ), and their growths were evaluated by measuring optical density (OD) at 600 nm with a spectrophotometer. Biochemical properties (oxidase, catalase, and the ability of carbon source usage) of isolates were performed according to the methods described by Harley and Prescott (2002) (Prescott 2002).

\section{Molecular identification of bacteria}

\section{Genomic DNA isolation}

Total genomic DNA was performed by modifying Sambrook's classic phenol: chloroform: isoamyl alcohol method (Sambrook 1987) and samples were stored at $-80^{\circ} \mathrm{C}$ (Prescott and Harley 2002).

\section{Cloning and sequencing of $16 \mathrm{~S}$ ribosomal RNA (16S rRNA) gene regions of isolates}

PCR amplification of bacterial isolates was conducted by using a GenePro Thermal Cycler (Bioer Technology Co., Ltd) TC-E-96G Model. In order to amplify the 16S rRNA gene region of DNA samples, universal primers (forward primer 27F = 5'-GAGTTTGATCCTGGCTCA-3'; reverse primer 1385R = 5'-

CGGTGTGT[A/G]CAAGGCCC-3') were used. Amplification reactions were carried out in $25 \mu \mathrm{L}$ volumes containing: 10X PCR buffer (Applied Biosystems, Roche, California, USA), $25 \mu \mathrm{M}$ magnesium chloride, $25 \mathrm{mM}$ dNTP, $25 \mathrm{pmol}$ of each primer, and 5 unit/ $\mu$ Taq DNA polymerase (Applied Biosystems). We performed the PCR under the following conditions: 1 cycle of $94^{\circ} \mathrm{C}$ for $5 \mathrm{~min}, 40$ cycles of $94^{\circ} \mathrm{C}$ for $1 \mathrm{~min}, 55^{\circ} \mathrm{C}$ for $1 \mathrm{~min}$ and $72^{\circ} \mathrm{C}$ for $1 \mathrm{~min}$ and $1 \mathrm{cycle}$ of $72^{\circ} \mathrm{C}$ for $5 \mathrm{~min}$. $1 \%$ agarose gel was prepared for visualization of PCR products by gel electrophoresis. The PCR products were purified, cloned, and sequenced according to the methods described by many researchers (Jacobus and Gross 2015, Li et al. 1999). The sequence data of all isolates were analyzed by using GenBank ('https://blast.ncbi.nlm.nih.gov/Blast.cgi') EzTaxon ('https://www.ezbiocloud.net/') server and the results were uploaded to the NCBI GenBank sequence database.

\section{Phylogenetic analysis}

The phylogenetic tree of the bacterial isolates was formed by using the neighbor-joining method with the MEGA 7 software (Oztas Gulmus and Gormez 2020b).

\section{Screening of enzyme activity of the bacterial isolates}

We screened all the isolates for enzyme activity to reveal their ability to produce extracellular hydrolase enzymes including amylase, lipase, cellulase, and protease.

Amylase Activity, All the isolates were inoculated on an agar plate containing $1 \%$ starch and amylase production after feeding with Lugol solution was determined as clear and large areas (Yadav et al. 2018).

Protease Activity, All the isolates were streaked on an agar medium containing $10 \%$ skimmed milk and the protease activity of the isolates that form a transparent zone on the agar plate was recorded as positive (Oztas Gulmus and Gormez 2020a).

Lipase Activity, All the isolates were streaked on an agar medium containing $1 \%$ tributyrin and the lipase activity of the isolates that form a hydrolysis zone on the agar plate was recorded as positive (Ertuğrul et al. 2007).

Cellulase Activity, All of the isolates were streaked on agar medium [carboxymethylcellulose $(10 \mathrm{~g} / \mathrm{L})$, peptone $(5 \mathrm{~g} / \mathrm{L}), \mathrm{yeast}(5 \mathrm{~g} / \mathrm{L}), \mathrm{KH}_{2} \mathrm{PO} \mathrm{O}_{4}(1 \mathrm{~g} / \mathrm{L})$, $\mathrm{MgSO}_{4} .7 \mathrm{H}_{2} \mathrm{O}(0.2 \mathrm{~g} / \mathrm{L}), \mathrm{NaCl}(10 \mathrm{~g} / \mathrm{L})$ and agar $\left.(15 \mathrm{~g} / \mathrm{L})\right]$. The cellulase activity of the isolates was determined by staining the Petri dishes with Congo Red Dye (Baltaci et al. 2017).

\section{Results}

The distribution of the hot springs in Turkey parallels the distribution of the fault systems and volcanic areas (Mutlu and Güleç 1998). The altitude of the studied area is between 1125 and 1925 meters above sea level. The source temperatures of the samples were measured as $38^{\circ} \mathrm{C}(\mathrm{Pasinler}), 60^{\circ} \mathrm{C}(\mathrm{Hista}$, 
Dargecit, and Guclukonak), and $75^{\circ} \mathrm{C}$ (Kopru, Davut, and Hasanabdal). Most of the sources were found to be at neutral pH, except for Hista, Köprü, and Davut hot springs ( $\mathrm{pH} 8.5$ and 9.1).

\section{The results of conventional test}

In the study, 83 bacterial isolates were got from Erzurum-Pasinler (26 isolates), Van-Hasanabdal (1 isolate), Siirt-Hista (6 isolates), Agri-Kopru (9 isolates), AgriDavut (37 isolates), Mardin-Dargecit ( 2 isolates), and Sirnak-Guclukonak (2 isolates) at different temperatures $\left(40^{\circ} \mathrm{C}, 55^{\circ} \mathrm{C}, 65^{\circ} \mathrm{C}, 70^{\circ} \mathrm{C}, 75^{\circ} \mathrm{C}, 85^{\circ} \mathrm{C}\right.$, and 90 ${ }^{\circ} \mathrm{C}$ ). According to the results of morphological tests, it was determined that all isolates were gram positive and rod-shaped, and formed endospores except for 9 isolates. Colonies of the isolates were cream, white and pink colors with rough, smooth, or shiny surfaces and smooth or irregular edges. The optimum temperature of isolated bacteria was determined as 55,60 , and $72^{\circ} \mathrm{C}$. Therefore, most of the isolates were considered as obligate thermophiles, which showed optimum growth at temperatures between $55-65^{\circ} \mathrm{C}$, whereas those that grew optimally at $50-55^{\circ} \mathrm{C}$ were defined as thermotolerant (Panosyan et al. 2020 ).

Therefore, most of the isolates (97.5\%) were being obligate thermophiles with optimum growth at temperatures between $55-65{ }^{\circ} \mathrm{C}$, while some isolates $(2.5 \%)$ that grow optimally at $50-55^{\circ} \mathrm{C}$ were recorded as heat resistant. Most isolates were shown optimum growth in the pH $5.0-9.5$ and $0-9 \% \mathrm{NaCl}$ concentration range. The results of the physiological tests revealed that most isolates could grow in high temperature and $\mathrm{pH}$ ranges (Table 1).

Table 1

The characteristic features of bacterial isolates 


\begin{tabular}{|c|c|c|c|c|c|c|c|c|c|}
\hline \multirow[b]{2}{*}{ Isolate Code } & \multicolumn{4}{|c|}{ Physiological Tests } & \multicolumn{5}{|c|}{ Morphological Tests } \\
\hline & $\mathrm{pH}$ & $\begin{array}{l}\mathrm{NaCl} \\
(\%)\end{array}$ & $\begin{array}{l}\text { Temperature } \\
\text { range } \mathrm{T}_{\text {opt }}\left({ }^{\circ} \mathrm{C}\right) / \\
\text { Temperature }\left({ }^{\circ} \mathrm{C}\right)\end{array}$ & $\begin{array}{l}\text { Anaerobic } \\
\text { growth }\end{array}$ & Movement & Morphology & Chain & Endospore & $\begin{array}{l}\text { Gram } \\
\text { test }\end{array}$ \\
\hline $\begin{array}{l}\text { BTX1, BTX2, BTX6, BTX7, ВTX8, BTX10, } \\
\text { BTX12, BTX13, BTX14, BTX23, BTX26, } \\
\text { BTX27, ВTX30, ВTX32, ВTX33, ВTX34, } \\
\text { BTX35 }\end{array}$ & $\begin{array}{l}5- \\
8,5\end{array}$ & $\leq 9$ & $40-85 / 72$ & + & + & Bacil & + & + & + \\
\hline $\begin{array}{l}\text { BTX3, BTX4, BTX5, BTX15, BTX22, } \\
\text { BTX28 }\end{array}$ & $\begin{array}{l}5- \\
8,5\end{array}$ & $\leq 9$ & $40-85 / 72$ & + & + & Bacil & - & + & + \\
\hline BTX25 & $\begin{array}{l}5- \\
8,5\end{array}$ & $\leq 9$ & $40-85 / 72$ & - & + & Bacil & - & - & + \\
\hline ВТX9, ВТX1, ВТX24, ВТХ31 & $\begin{array}{l}5- \\
8,5\end{array}$ & $\leq 9$ & $40-85 / 72$ & - & + & Bacil & - & + & + \\
\hline BTX16 & $\begin{array}{l}5- \\
8,5\end{array}$ & $\leq 5$ & $40-85 / 72$ & + & + & Coco Bacil & - & + & + \\
\hline ВTX17, ВТХ36 & $\begin{array}{l}5- \\
8,5\end{array}$ & $\leq 5$ & $40-85 / 72$ & + & + & Bacil & - & + & + \\
\hline BTX18 & $\begin{array}{l}5- \\
8,5\end{array}$ & $\leq 5$ & $40-85 / 72$ & + & + & Bacil & - & - & + \\
\hline BTX19 & $\begin{array}{l}5- \\
8,5\end{array}$ & $\leq 5$ & $40-85 / 72$ & - & + & Bacil & + & + & + \\
\hline BTX20, BTX21 & $\begin{array}{l}5- \\
8,5\end{array}$ & $\leq 5$ & $40-85 / 72$ & + & + & Bacil & + & + & + \\
\hline BTX29 & $\begin{array}{l}5- \\
7,5\end{array}$ & $\leq 5$ & $40-85 / 72$ & + & + & Bacil & + & + & + \\
\hline ВTX37 & $\begin{array}{l}5- \\
7,5\end{array}$ & $<5$ & $40-75 / 60$ & - & + & Bacil & - & + & + \\
\hline ВТX38 & $\begin{array}{l}5- \\
7,5\end{array}$ & $<5$ & $40-75 / 60$ & + & + & Bacil & - & + & + \\
\hline ВТX39 & $\begin{array}{l}5- \\
7,5\end{array}$ & $<5$ & $40-75 / 60$ & + & + & Bacil & + & + & + \\
\hline BTX40, BTX41 & $\begin{array}{l}5- \\
7,5\end{array}$ & $<5$ & $40-75 / 60$ & + & + & Bacil & + & + & + \\
\hline BTX42, BTX50 & $\begin{array}{l}5- \\
7,5\end{array}$ & $<7$ & $40-75 / 60$ & + & + & Bacil & - & + & + \\
\hline BTX43 & $\begin{array}{l}5- \\
7,5\end{array}$ & $<7$ & $40-75 / 60$ & - & + & Bacil & + & + & + \\
\hline BTX44 & $\begin{array}{l}5- \\
7,5\end{array}$ & $<7$ & $40-75 / 60$ & - & + & Bacil & - & + & + \\
\hline BTX45, BTX46, ВTX47 & $\begin{array}{l}5- \\
7,5\end{array}$ & $<7$ & $40-75 / 60$ & + & + & Bacil & + & + & + \\
\hline BTX48, ВTX78 & $\begin{array}{l}5- \\
7,5\end{array}$ & $\leq 9$ & $40-75 / 60$ & - & + & Bacil & - & - & + \\
\hline BTX49 & $\begin{array}{l}5- \\
7,5\end{array}$ & $<7$ & $40-75 / 60$ & + & + & Bacil & - & - & + \\
\hline BTX51 & $\begin{array}{l}5- \\
7,5\end{array}$ & $<7$ & $40-85 / 72$ & + & + & Bacil & - & - & + \\
\hline BTX52 & $\begin{array}{l}5- \\
7,5\end{array}$ & $<7$ & $40-85 / 72$ & - & + & Bacil & - & + & + \\
\hline BTX53 & $\begin{array}{l}6- \\
9,5\end{array}$ & $<7$ & $40-85 / 72$ & + & + & Bacil & + & + & + \\
\hline BTX54 & $\begin{array}{l}6- \\
9,5\end{array}$ & $<7$ & $40-85 / 72$ & + & + & Bacil & + & + & + \\
\hline BTX55 & $\begin{array}{l}6- \\
9,5\end{array}$ & $<7$ & $40-85 / 72$ & - & + & Bacil & - & + & + \\
\hline BTX56 & $\begin{array}{l}6- \\
7,5\end{array}$ & $<7$ & $40-70 / 55$ & + & + & Bacil & + & + & + \\
\hline BTX57 & $\begin{array}{l}6- \\
7,5\end{array}$ & $<7$ & $40-70 / 55$ & + & + & Bacil & - & + & + \\
\hline
\end{tabular}




\begin{tabular}{|c|c|c|c|c|c|c|c|c|c|}
\hline BTX58 & $\begin{array}{l}5- \\
7,5\end{array}$ & $<7$ & $40-75 / 60$ & - & + & Bacil & - & - & + \\
\hline BTX59 & $\begin{array}{l}5- \\
7,5\end{array}$ & $<7$ & $40-75 / 60$ & + & + & Bacil & + & + & + \\
\hline BTX60 & $\begin{array}{l}6- \\
9,5\end{array}$ & $\leq 9$ & $40-85 / 72$ & + & + & Bacil & + & + & + \\
\hline BTX61 & $\begin{array}{l}6- \\
9,5\end{array}$ & $\leq 9$ & $40-85 / 72$ & - & + & Bacil & - & - & + \\
\hline ВTX62, ВTX63 & $\begin{array}{l}5- \\
8,5\end{array}$ & $\leq 6$ & $40-85 / 72$ & + & + & Bacil & + & + & + \\
\hline BTX64 & $\begin{array}{l}5- \\
8,5\end{array}$ & $\leq 6$ & $40-85 / 72$ & + & + & Bacil & - & + & + \\
\hline ВTX65, ВTX66, ВТX67, ВТХ68 & $\begin{array}{l}5- \\
7,5\end{array}$ & $\leq 6$ & $40-85 / 72$ & + & + & Bacil & + & + & + \\
\hline BTX69, BTX71 & $\begin{array}{l}5,5- \\
7,5\end{array}$ & $<7$ & $40-75 / 60$ & + & + & Bacil & - & + & + \\
\hline ВТX70, ВТX72, ВТX73 & $\begin{array}{l}5,5- \\
7,5\end{array}$ & $<7$ & $40-75 / 60$ & + & + & Bacil & + & + & + \\
\hline BTX74 & $\begin{array}{l}5,5- \\
7,5\end{array}$ & $<6$ & $40-75 / 60$ & + & + & Bacil & + & + & + \\
\hline BTX75, ВTX76 & $\begin{array}{l}5,5- \\
7,5\end{array}$ & $<6$ & $40-75 / 60$ & + & + & Bacil & - & + & + \\
\hline BTX77 & $\begin{array}{l}6- \\
7,5\end{array}$ & $<7$ & $40-75 / 60$ & + & + & Bacil & - & - & + \\
\hline BTX79 & $5-8$ & $<7$ & $40-85 / 72$ & + & + & Bacil & + & + & + \\
\hline BTX80 & $6,7,5$ & $<6$ & $40-85 / 72$ & + & + & Bacil & + & + & + \\
\hline BTX81 & $\begin{array}{l}5- \\
7,5\end{array}$ & $\leq 9$ & $40-85 / 72$ & + & + & Bacil & + & + & + \\
\hline BTX82 & $\begin{array}{l}5- \\
7,5\end{array}$ & $<7$ & $40-85 / 72$ & + & + & Bacil & + & + & + \\
\hline BTX83 & $\begin{array}{l}5- \\
7,5\end{array}$ & $<6$ & $40-85 / 72$ & + & + & Bacil & + & + & + \\
\hline
\end{tabular}

\section{The results of 16s rRNA sequencing analyses of bacterial isolates}

16S rRNA gene regions were amplified with universal primers and visualized by gel electrophoresis. According to the results of the blast analysis, it was determined that all isolates contained 862-1419 base pair nucleotides (Table 2). All bacteria showing thermophilic properties were closely related to members of 2 different genera, Bacillus and Geobacillus (Fig. 2). Sequence analysis identified 68 strains of Bacillus (81.93\%) and 15 strains of Geobacillus (18.07\%). According to the results of blast analysis, the closest match (100\% similarity) to isolate (BTX53-59, BTX72-73, and BTX79) was with B. thuringiensis strain KNU-07, which was isolated from Korea. Based on the 16S rRNA gene sequence similarity analysis, 33 isolates demonstrated 95 to $99 \%$ sequence similarity to species $B$. subtilis. Among the described species, the closest relatives of isolates BTX42-47, BTX49-52, BTX69-71, BTX77, and BTX78 were Geobacilluskaustophilus with a sequence homology rate of 99\%. Among the described species, the closest relatives of isolates BTX16-19, BTX20, BTX21, BTX29, BTX36-41, BTX72, BTX73, and BTX79 were B. licheniformis with a sequence homology rate of $97-99 \%$. Among the described species, the closest relatives of isolates BTX62-68, BTX74-76, and BTX83 were B. coagulans with a sequence homology rate of $93-98 \%$.

\section{The results of phylogenetic analysis}

The phylogenetic tree was created with $16 \mathrm{~S}$ gene sequencing data of identified bacteria, their type strains, and outgroup to determine the evolutionary relationships (Fig. 3). The evolutionary relationship was inferred using the Neighbor-Joining method (Saitou and Nei 1987). The optimal tree with the sum of branch length $=0.34395833$ was shown. The percentage of replicate trees in which the associated taxa clustered together in the bootstrap test (1000 replicates) is shown next to the branches (Felsenstein 1985). The tree was drawn to scale, with branch lengths in the same units as those of the evolutionary distances used to infer the phylogenetic tree. The evolutionary distances were computed by using the p-distance method (Chakraborty 2002) and are in the units of the number of base differences per site. The analysis of 89 nucleotide sequences (83 isolates, 5 type strains, and 1 outgroup) was conducted in MEGA7 (Kumar et al. 2016). As shown in the figure, bacteria belonging to the genus Bacillus were divided into distinct groups from the genus Geobacillus, to be a single group with type strains as expected. The results showed that there was no important difference between the groups and the bacteria in terms of locations or optimum temperature of the isolates. 
BLAST results and accession numbers of 16S rRNA gene sequences of isolates

Page 7/19 


\begin{tabular}{|c|c|c|c|c|c|}
\hline Isolate Code & Identification & GenBank Number & Similarity $^{*}$ rate (\%) & $\begin{array}{l}\text { Base length }^{\star \star} \text { (nucleotide) } \\
\text { (nucleotide) }\end{array}$ & Location \\
\hline BTX1 & Bacillus subtilis & MH101281 & $99 \%$ & 1397 & Davut \\
\hline BTX2 & Bacillus subtilis & MH101282 & $99 \%$ & 1387 & Davut \\
\hline ВTX3 & Bacillus subtilis & MH101283 & $99 \%$ & 1385 & Davut \\
\hline BTX4 & Bacillus subtilis & МH101284 & $99 \%$ & 1389 & Davut \\
\hline BTX5 & Bacillus subtilis & MH101285 & $99 \%$ & 1386 & Davut \\
\hline BTX6 & Bacillus subtilis & MH101286 & $99 \%$ & 1384 & Davut \\
\hline BTX7 & Bacillus subtilis & MH101287 & $99 \%$ & 1382 & Davut \\
\hline BTX8 & Bacillus subtilis & MH101288 & $99 \%$ & 1381 & Davut \\
\hline BTX9 & Bacillus subtilis & MH101289 & $99 \%$ & 1386 & Davut \\
\hline BTX10 & Bacillus subtilis & MH101290 & $99 \%$ & 1386 & Davut \\
\hline BTX11 & Bacillus subtilis & MH101291 & $99 \%$ & 1380 & Davut \\
\hline BTX12 & Bacillus subtilis & MH101292 & $99 \%$ & 1386 & Davut \\
\hline BTX13 & Bacillus subtilis & MH101293 & $99 \%$ & 1385 & Davut \\
\hline BTX14 & Bacillus subtilis & MH101294 & $99 \%$ & 1383 & Davut \\
\hline BTX15 & Bacillus subtilis & MH101295 & $99 \%$ & 1387 & Davut \\
\hline BTX16 & Bacillus licheniformis & MH101314 & $98 \%$ & 1417 & Davut \\
\hline BTX17 & Bacillus licheniformis & MH101315 & $98 \%$ & 1410 & Davut \\
\hline BTX18 & Bacillus licheniformis & MH101316 & $98 \%$ & 1411 & Davut \\
\hline BTX19 & Bacillus licheniformis & MH101317 & $98 \%$ & 1414 & Davut \\
\hline BTX20 & Bacillus licheniformis & MH101318 & $98 \%$ & 1413 & Davut \\
\hline BTX21 & Bacillus licheniformis & MH101319 & $98 \%$ & 1416 & Davut \\
\hline BTX22 & Bacillus subtilis & MH101296 & $99 \%$ & 1384 & Davut \\
\hline BTX23 & Bacillus subtilis & MH101297 & $99 \%$ & 1384 & Davut \\
\hline BTX24 & Bacillus subtilis & MH101298 & $99 \%$ & 1384 & Davut \\
\hline BTX25 & Bacillus subtilis & MH101299 & $99 \%$ & 1387 & Davut \\
\hline BTX26 & Bacillus subtilis & MH101300 & $99 \%$ & 1384 & Davut \\
\hline BTX27 & Bacillus subtilis & MH101301 & $99 \%$ & 1383 & Davut \\
\hline BTX28 & Bacillus subtilis & MH101302 & $99 \%$ & 1386 & Davut \\
\hline BTX29 & Bacillus licheniformis & MH101320 & $98 \%$ & 1410 & Davut \\
\hline ВTX30 & Bacillus subtilis & MH101303 & $99 \%$ & 1384 & Davut \\
\hline BTX31 & Bacillus subtilis & MH101304 & $99 \%$ & 1384 & Davut \\
\hline BTX32 & Bacillus subtilis & MH101305 & $99 \%$ & 1384 & Davut \\
\hline ВТX33 & Bacillus subtilis & MH101306 & $99 \%$ & 1391 & Davut \\
\hline ВTX34 & Bacillus subtilis & MH101307 & $99 \%$ & 1380 & Davut \\
\hline BTX35 & Bacillus subtilis & MH101308 & $99 \%$ & 1387 & Davut \\
\hline BTX36 & Bacillus licheniformis & MH101321 & $98 \%$ & 1410 & Davut \\
\hline BTX37 & Bacillus licheniformis & MH101322 & $98 \%$ & 1413 & Pasinler \\
\hline BTX38 & Bacillus licheniformis & MH101323 & $98 \%$ & 1419 & Pasinler \\
\hline BTX39 & Bacillus licheniformis & MH101324 & $98 \%$ & 1415 & Pasinler \\
\hline BTX40 & Bacillus licheniformis & MH101325 & $98 \%$ & 1418 & Pasinler \\
\hline BTX41 & Bacillus licheniformis & MH101326 & $98 \%$ & 1411 & Pasinler \\
\hline
\end{tabular}




\begin{tabular}{|c|c|c|c|c|c|}
\hline BTX42 & Geobacillus kaustophilus & MH101338 & $98 \%$ & 1415 & Pasinler \\
\hline BTX43 & Geobacillus kaustophilus & MH101339 & $98 \%$ & 1411 & Pasinler \\
\hline BTX44 & Geobacillus kaustophilus & MH101340 & $98 \%$ & 1410 & Pasinler \\
\hline BTX45 & Geobacillus kaustophilus & MH101341 & $98 \%$ & 1409 & Pasinler \\
\hline BTX46 & Geobacillus kaustophilus & MH101342 & $98 \%$ & 1418 & Pasinler \\
\hline BTX47 & Geobacillus kaustophilus & MH101343 & $98 \%$ & 1410 & Pasinler \\
\hline BTX48 & Bacillus subtilis & MH101309 & $98 \%$ & 1384 & Pasinler \\
\hline BTX49 & Geobacillus kaustophilus & MH101344 & $98 \%$ & 1410 & Pasinler \\
\hline BTX50 & Geobacillus kaustophilus & MH101345 & $98 \%$ & 1414 & Pasinler \\
\hline BTX51 & Geobacillus kaustophilus & MH101346 & $98 \%$ & 1412 & Pasinler \\
\hline BTX52 & Geobacillus kaustophilus & MH101347 & $98 \%$ & 1410 & Hista \\
\hline BTX53 & Bacillus thuringiensis & MH101328 & $100 \%$ & 873 & Hista \\
\hline BTX54 & Bacillus thuringiensis & MH101329 & $100 \%$ & 868 & Hista \\
\hline BTX55 & Bacillus thuringiensis & MH101330 & $100 \%$ & 866 & Hista \\
\hline BTX56 & Bacillus thuringiensis & MH101331 & $100 \%$ & 866 & Guclukonak \\
\hline BTX57 & Bacillus thuringiensis & MH101332 & $100 \%$ & 866 & Guclukonak \\
\hline BTX58 & Bacillus thuringiensis & MH101333 & $100 \%$ & 878 & Dargecit \\
\hline BTX59 & Bacillus thuringiensis & MH101334 & $100 \%$ & 864 & Dargecit \\
\hline BTX60 & Bacillus subtilis & MH101310 & $99 \%$ & 1384 & Hista \\
\hline BTX61 & Bacillus subtilis & MH101311 & $99 \%$ & 1384 & Hista \\
\hline BTX62 & Bacillus coagulans & MH101353 & $98 \%$ & 1339 & Davut \\
\hline BTX63 & Bacillus coagulans & MH101354 & $98 \%$ & 1352 & Kopru \\
\hline BTX64 & Bacillus coagulans & MH101355 & $98 \%$ & 1343 & Kopru \\
\hline BTX65 & Bacillus coagulans & MH101356 & $98 \%$ & 1346 & Kopru \\
\hline BTX66 & Bacillus coagulans & MH101357 & $98 \%$ & 1347 & Hasanabda \\
\hline BTX67 & Bacillus coagulans & MH101358 & $98 \%$ & 1343 & Kopru \\
\hline BTX68 & Bacillus coagulans & MH101359 & $98 \%$ & 1343 & Pasinler \\
\hline BTX69 & Geobacillus kaustophilus & MH101348 & $98 \%$ & 1408 & Pasinler \\
\hline BTX70 & Geobacillus kaustophilus & MH101349 & $98 \%$ & 1410 & Pasinler \\
\hline BTX71 & Geobacillus kaustophilus & MH101350 & $98 \%$ & 1413 & Pasinler \\
\hline BTX72 & Bacillus thuringiensis & MH101335 & $100 \%$ & 867 & Pasinler \\
\hline BTX73 & Bacillus thuringiensis & MH101336 & $100 \%$ & 862 & Pasinler \\
\hline BTX74 & Bacillus coagulans & MH101360 & $98 \%$ & 1355 & Pasinler \\
\hline BTX75 & Bacillus coagulans & MH101361 & $98 \%$ & 1341 & Pasinler \\
\hline BTX76 & Bacillus coagulans & MH101362 & $98 \%$ & 1350 & Pasinler \\
\hline BTX77 & Geobacillus kaustophilus & MH101351 & $98 \%$ & 1410 & Pasinler \\
\hline BTX78 & Bacillus subtilis & MH101312 & $99 \%$ & 1394 & Pasinler \\
\hline BTX79 & Bacillus thuringiensis & MH101337 & $100 \%$ & 867 & Kopru \\
\hline BTX80 & Geobacillus kaustophilus & MH101352 & $98 \%$ & 1408 & Kopru \\
\hline BTX81 & Bacillus subtilis & MH101313 & $99 \%$ & 1387 & Kopru \\
\hline BTX82 & Bacillus licheniformis & MH101327 & $98 \%$ & 1409 & Kopru \\
\hline BTX83 & Bacillus coagulans & MH101363 & $98 \%$ & 1343 & Kopru \\
\hline
\end{tabular}




\section{Determination of enzyme production ability of bacterial isolate}

In the study, the ability of all isolates was examined to produce amylase, protease, cellulose, and lipase enzymes (Table 3$)$. According to the qualitative analysis results, it was determined that 27 (32.8\%) isolates can produce amylase, protease, cellulase, and lipase (Fig. 4). In the study, 68 (82\%), 73 (88\%), 34 $(41 \%)$, and $69(83 \%)$ isolates were determined as producers of amylase, protease, cellulase, and lipase, respectively. Among the isolates, BTX55 had only protease activity, BTX18 and BTX27 had lipase, BTX9 isolate had amylase activity, while all the remaining isolates had over one enzyme activity (Fig 3).

Table 3

Biochemical characteristics and enzyme profiles of bacterial isolates 


\begin{tabular}{|c|c|c|c|c|c|c|c|c|c|c|c|c|c|}
\hline \multirow[b]{2}{*}{$\begin{array}{l}\text { Isolate } \\
\text { Code }\end{array}$} & \multirow[b]{2}{*}{ Identification } & \multicolumn{2}{|c|}{$\begin{array}{l}\text { Biochemical } \\
\text { characteristics }\end{array}$} & \multicolumn{7}{|c|}{ Carbon source } & \multicolumn{3}{|c|}{ Enzyme activities } \\
\hline & & Catalase & Oxidase & Glucose & Lactose & Galactose & Fructose & Citrate & Casein & Starch & Cellulase & Lipase & Prot \\
\hline BTX1 & $\begin{array}{l}\text { Bacillus } \\
\text { subtilis }\end{array}$ & + & - & + & - & nd & + & nd & + & + & - & + & + \\
\hline BTX2 & $\begin{array}{l}\text { Bacillus } \\
\text { subtilis }\end{array}$ & + & + & + & + & + & + & + & - & - & + & + & + \\
\hline BTX3 & $\begin{array}{l}\text { Bacillus } \\
\text { subtilis }\end{array}$ & + & + & + & - & - & - & - & - & + & - & + & + \\
\hline BTX4 & $\begin{array}{l}\text { Bacillus } \\
\text { subtilis }\end{array}$ & - & + & - & - & + & - & - & + & + & - & - & + \\
\hline BTX5 & $\begin{array}{l}\text { Bacillus } \\
\text { subtilis }\end{array}$ & + & - & + & - & + & - & - & - & - & - & + & + \\
\hline BTX6 & $\begin{array}{l}\text { Bacillus } \\
\text { subtilis }\end{array}$ & + & + & + & + & + & + & + & - & + & + & + & + \\
\hline BTX7 & $\begin{array}{l}\text { Bacillus } \\
\text { subtilis }\end{array}$ & + & + & - & + & - & + & - & - & + & + & - & + \\
\hline BTX8 & $\begin{array}{l}\text { Bacillus } \\
\text { subtilis }\end{array}$ & + & + & + & + & - & + & - & + & + & + & + & + \\
\hline BTX9 & $\begin{array}{l}\text { Bacillus } \\
\text { subtilis }\end{array}$ & - & - & - & - & + & - & - & + & + & - & - & - \\
\hline BTX10 & $\begin{array}{l}\text { Bacillus } \\
\text { subtilis }\end{array}$ & + & + & + & + & + & + & + & + & + & + & + & + \\
\hline BTX11 & $\begin{array}{l}\text { Bacillus } \\
\text { subtilis }\end{array}$ & + & + & + & - & - & - & - & - & - & - & + & + \\
\hline BTX12 & $\begin{array}{l}\text { Bacillus } \\
\text { subtilis }\end{array}$ & + & + & + & + & + & + & + & + & + & + & + & + \\
\hline BTX13 & $\begin{array}{l}\text { Bacillus } \\
\text { subtilis }\end{array}$ & - & + & - & + & - & + & - & + & + & + & + & + \\
\hline BTX14 & $\begin{array}{l}\text { Bacillus } \\
\text { subtilis }\end{array}$ & + & + & + & + & + & + & - & - & + & + & - & - \\
\hline BTX15 & $\begin{array}{l}\text { Bacillus } \\
\text { subtilis }\end{array}$ & - & + & + & - & - & - & - & - & + & - & + & + \\
\hline BTX16 & $\begin{array}{l}\text { Bacillus } \\
\text { licheniformis }\end{array}$ & + & + & + & - & + & - & - & - & - & - & + & + \\
\hline BTX17 & $\begin{array}{l}\text { Bacillus } \\
\text { licheniformis }\end{array}$ & + & + & + & - & - & - & - & + & + & - & + & + \\
\hline BTX18 & $\begin{array}{l}\text { Bacillus } \\
\text { licheniformis }\end{array}$ & - & nd & - & nd & + & - & nd & - & + & - & + & - \\
\hline BTX19 & $\begin{array}{l}\text { Bacillus } \\
\text { licheniformis }\end{array}$ & + & + & + & + & + & + & + & + & + & - & - & + \\
\hline BTX20 & $\begin{array}{l}\text { Bacillus } \\
\text { licheniformis }\end{array}$ & + & + & + & + & - & + & + & + & + & + & + & + \\
\hline BTX21 & $\begin{array}{l}\text { Bacillus } \\
\text { licheniformis }\end{array}$ & - & + & - & + & + & + & - & - & - & - & + & + \\
\hline BTX22 & $\begin{array}{l}\text { Bacillus } \\
\text { subtilis }\end{array}$ & - & + & - & + & + & - & - & + & + & + & + & - \\
\hline BTX23 & $\begin{array}{l}\text { Bacillus } \\
\text { subtilis }\end{array}$ & + & + & + & + & - & + & + & + & + & + & + & + \\
\hline BTX24 & $\begin{array}{l}\text { Bacillus } \\
\text { subtilis }\end{array}$ & + & + & - & - & - & + & - & + & + & - & + & + \\
\hline BTX25 & $\begin{array}{l}\text { Bacillus } \\
\text { subtilis }\end{array}$ & - & - & - & - & + & - & - & - & + & - & + & + \\
\hline BTX26 & $\begin{array}{l}\text { Bacillus } \\
\text { subtilis }\end{array}$ & + & + & nd & + & - & + & + & + & - & - & - & + \\
\hline BTX27 & $\begin{array}{l}\text { Bacillus } \\
\text { subtilis }\end{array}$ & + & + & + & - & - & + & - & + & + & - & + & - \\
\hline BTX28 & Bacillus & + & + & - & - & + & - & - & + & + & - & + & + \\
\hline
\end{tabular}


subtilis

licheniformis

BTX30 Bacillus

subtilis

BTX31 Bacillus

subtilis

BTX32 Bacillus

subtilis

BTX33 Bacillus

subtilis

BTX34 Bacillus

subtilis

BTX35 $\begin{aligned} & \text { Bacillus } \\ & \text { subtilis }\end{aligned}$

BTX36 Bacillus

licheniformis

BTX37 Bacillus

licheniformis

BTX38 Bacillus

licheniformis

BTX39 Bacillus

licheniformis

BTX40 Bacillus

licheniformis

BTX41 Bacillus

licheniformis

BTX42 Geobacillus

kaustophilus

BTX43 Geobacillus

kaustophilus

BTX44 Geobacillus

kaustophilus

BTX45 Geobacillus

kaustophilus

BTX46 Geobacillus

kaustophilus

BTX47 Geobacillus

kaustophilus

BTX48 $\begin{aligned} & \text { Bacillus } \\ & \text { subtilis }\end{aligned} \quad+$

BTX49 Geobacillus - nd

kaustophilus

BTX50 Geobacillus

kaustophilus

BTX51 Geobacillus

kaustophilus

BTX52 Geobacillus kaustophilus

BTX53 Bacillus thuringiensis

BTX54 Bacillus thuringiensis

BTX55 Bacillus thuringiensis

BTX56 Bacillus

thuringiensis

BTX57 Bacillus

thuringiensis 


\begin{tabular}{|c|c|c|c|c|c|c|c|c|c|c|c|c|c|}
\hline BTX58 & $\begin{array}{l}\text { Bacillus } \\
\text { thuringiensis }\end{array}$ & - & + & - & nd & + & - & - & - & - & - & + & + \\
\hline BTX59 & $\begin{array}{l}\text { Bacillus } \\
\text { thuringiensis }\end{array}$ & + & + & + & + & + & + & + & + & + & + & + & + \\
\hline BTX60 & $\begin{array}{l}\text { Bacillus } \\
\text { subtilis }\end{array}$ & + & + & + & + & - & + & + & + & + & + & + & + \\
\hline BTX61 & $\begin{array}{l}\text { Bacillus } \\
\text { subtilis }\end{array}$ & - & - & + & - & + & - & - & - & + & - & + & + \\
\hline BTX62 & $\begin{array}{l}\text { Bacillus } \\
\text { coagulans }\end{array}$ & - & + & + & + & + & nd & + & + & - & - & + & + \\
\hline BTX63 & $\begin{array}{l}\text { Bacillus } \\
\text { coagulans }\end{array}$ & + & + & + & + & + & + & + & + & + & + & + & + \\
\hline BTX64 & $\begin{array}{l}\text { Bacillus } \\
\text { coagulans }\end{array}$ & + & + & - & - & - & + & - & + & + & - & + & + \\
\hline BTX65 & $\begin{array}{l}\text { Bacillus } \\
\text { coagulans }\end{array}$ & + & + & + & + & + & + & - & + & + & + & + & + \\
\hline BTX66 & $\begin{array}{l}\text { Bacillus } \\
\text { coagulans }\end{array}$ & - & + & + & + & + & + & - & + & - & - & + & + \\
\hline BTX67 & $\begin{array}{l}\text { Bacillus } \\
\text { coagulans }\end{array}$ & + & - & + & + & + & + & + & + & + & + & + & + \\
\hline BTX68 & $\begin{array}{l}\text { Bacillus } \\
\text { coagulans }\end{array}$ & + & - & + & + & + & + & + & + & + & + & + & + \\
\hline BTX69 & $\begin{array}{l}\text { Geobacillus } \\
\text { kaustophilus }\end{array}$ & - & - & + & nd & + & + & nd & + & - & - & + & + \\
\hline BTX70 & $\begin{array}{l}\text { Geobacillus } \\
\text { kaustophilus }\end{array}$ & + & + & + & + & - & + & + & + & - & + & + & + \\
\hline BTX71 & $\begin{array}{l}\text { Geobacillus } \\
\text { kaustophilus }\end{array}$ & + & + & + & - & + & - & - & + & + & - & + & + \\
\hline BTX72 & $\begin{array}{l}\text { Bacillus } \\
\text { thuringiensis }\end{array}$ & - & + & - & + & - & + & + & + & + & - & + & + \\
\hline BTX73 & $\begin{array}{l}\text { Bacillus } \\
\text { thuringiensis }\end{array}$ & + & + & + & + & + & + & - & + & - & + & + & + \\
\hline BTX74 & $\begin{array}{l}\text { Bacillus } \\
\text { coagulans }\end{array}$ & + & + & + & + & + & + & + & + & + & + & + & + \\
\hline BTX75 & $\begin{array}{l}\text { Bacillus } \\
\text { coagulans }\end{array}$ & + & + & + & - & - & + & - & + & + & - & + & + \\
\hline BTX76 & $\begin{array}{l}\text { Bacillus } \\
\text { coagulans }\end{array}$ & + & + & + & - & + & - & - & + & + & + & + & + \\
\hline BTX77 & $\begin{array}{l}\text { Geobacillus } \\
\text { kaustophilus }\end{array}$ & + & - & - & - & + & - & - & - & + & + & - & + \\
\hline BTX78 & $\begin{array}{l}\text { Bacillus } \\
\text { subtilis }\end{array}$ & - & + & nd & nd & + & - & nd & - & - & - & + & + \\
\hline BTX79 & $\begin{array}{l}\text { Bacillus } \\
\text { thuringiensis }\end{array}$ & - & + & + & + & + & + & + & + & + & - & + & + \\
\hline BTX80 & $\begin{array}{l}\text { Geobacillus } \\
\text { kaustophilus }\end{array}$ & nd & - & nd & nd & + & + & + & + & - & + & - & - \\
\hline BTX81 & $\begin{array}{l}\text { Bacillus } \\
\text { subtilis }\end{array}$ & + & + & + & + & + & + & - & - & - & + & + & - \\
\hline BTX82 & $\begin{array}{l}\text { Bacillus } \\
\text { licheniformis }\end{array}$ & + & + & + & + & - & + & - & + & + & - & + & + \\
\hline BTX83 & $\begin{array}{l}\text { Bacillus } \\
\text { coagulans }\end{array}$ & + & - & + & + & - & + & - & + & + & - & + & - \\
\hline
\end{tabular}

$(+)$; observed reaction, (-); negative reaction, (nd); not detected

\section{Discussion}


The hot springs investigated in the present study are in tectonically active regions of the Eastern and Southeastern Anatolia regions of Turkey. 83 bacterial isolates with growth potential at $40^{\circ} \mathrm{C}$ and $75^{\circ} \mathrm{C}$ were got from Davut (37 isolates), Pasinler (26 isolates), Kopru (9 isolates), and Hasanabdal ( 1 isolate), and coded in sequential order from BTX1 to BTX83. Sequence analysis of all isolated bacteria was performed by using 16S rRNA gene regions. Because of the BLAST analysis of the obtained isolates, it was revealed that there were $98-100 \%$ similarities with their closest matches. Although similar studies have been conducted to isolate different thermophilic bacteria species in the hot springs in these regions, different species have been detected in hot springs such as Hasanabdal, Kopru, Pasinler, and Hista. According to the results of the study, B. subtilis (33), G. kaustophilus (15), B. licheniformis (14 isolates), B. coagulans (11), and $B$. thuringiensis (10) were identified as most common bacilli in the area. Amongst the isolated bacteria, Bacillus was the most dominant genus isolated from hot springs in this study. Studies conducted by many researchers have also shown that the microbial diversity studied in the hot springs reports the abundance and diversity of Bacillus species (Abdollahi et al. 2021, Baltaci et al. 2017, Ifandi and Alwi 2018, Margaryan et al. 2018, Oztas Gulmus and Gormez 2020b, Panosyan et al. 2020). Although the locations of Davut and Kopru hot springs are almost very close to each other, the isolated bacteria were quite different. B. subtilis and B. licheniformis were isolated from Davut, B. coagulans were also isolated from Kopru. The locations of other hot springs differ completely from each other. $B$. coagulans and $B$. thuringiensis were isolated from Hista for the first time. The $B$. coagulans were isolated for the first time from Diyadin and Hasanabdal. Geobacillus sp. and B. licheniformis were reported from Hasanabdal in similar studies (Poddar and Das 2018). Besides, the strains identified within the study (B. subtilis, Geobacillus kaustophilus, B. licheniformis, B. coagulans and B. thuringiensis) exhibit similar morphological, characteristic, physiological, and biochemical properties when compared with the literature (Baltaci et al. 2017, Oztas Gulmus and Gormez 2020a). All the isolated strains (B. coagulans, B. thuringiensis, B. subtilis, Geobacillus sp. and B. licheniformis) exhibited the ability to grow normally at high temperatures and $\mathrm{pH}$ values, which is not typical. The strains could grow at temperatures ranging from 40 to $85^{\circ} \mathrm{C}, \mathrm{NaCl}$ levels up to $9 \%(\mathrm{w} / \mathrm{v})$, and $\mathrm{pH}$ ranges from 4 to 9 . Only strains belonging to $B$. licheniformis isolates were able to grow in $\mathrm{NaCl}$ levels below $5 \%$ at $40-75^{\circ} \mathrm{C}$ (optimum at $60^{\circ} \mathrm{C}$ ) and pH 4 to $9(\mathrm{optimum}$ at pH 7 ).

Microbial enzymes, especially enzymes derived from extremophile organisms, are widely used in many areas today. Thermophilic organisms are also microorganisms that adapt to extreme conditions, so they can create a variety of products that can be used even in unfavorable conditions. Many enzymes from thermophilic organisms such as cellulase, xylanase, catalase, lactase, lipase, sucrase, pullulanase, pectinase, amylase, and protease are purified and produced commercially. These enzymes are widely used in many fields such as textile, food, detergent, industry, beverage industry, and health (Oztas Gulmus and Gormez 2020b). Bacillus species, which can be isolated from many environments as well as hot water sources, are of great importance in terms of enzyme production. Bacillus species are one of the most effective sources of proteolytic and carbohydrates. It meets the enzyme needs especially in many areas such as starch, bread, fruit juice, paper bleaching, and brewing. For this reason, many enzymes synthesized by Bacillus species are used in many areas in the industry (Oztas Gulmus and Gormez 2020b). The bacteria isolated in this study can be evaluated in a helpful position in future studies because of their thermophilic properties, being highly stable enzymes that can operate at high temperatures and extreme conditions.

\section{Conclusions}

This study has documented the isolated and identified bacteria in seven hot springs from Eastern and Southeastern Anatolia regions using 16S rDNA sequencing. According to $16 \mathrm{~S}$ rDNA sequencing results, most of the sequences were observed to belong to the genera Bacillus (81.93\%) and Geobacillus (18.07\%). These results suggest that all the isolates were Firmicutes and Bacillus was the most dominant genus in seven hot springs. Although the isolated bacteria from different seven hot springs were similar, they were quite diverse in their environmental attributes, encompassing a temperature range from 40 to $85^{\circ} \mathrm{C}, \mathrm{pH}$ ranges from 4 to 9 , and salinity from 0 to $9 \%$. Because of these important properties, wide $\mathrm{pH}$ and temperature ranges, these enzymes can be preferred in industrial applications. In this context, the presented study is designed as a preliminary study showing the diversity of bacteria that produce crucial thermophile enzymes in industrial and biotechnological terms.

\section{Declarations}

\section{Acknowledgments}

We thank Prof. Dr. Muhammed Sakiroglu for helping proof reading.

It presented the species identification phase of the study in 2017 as an international congress summary.

\section{Author contributions}

OU, Conceptualization, Methodology, Validation, Investigation, Resources, Formal analysis, Investigation, Resources, Writing - Original Draft, Writing - Review \& Editing; AG, Methodology, Investigation, Resources, Writing - Review \& Editing, Supervision. CO, Conceptualization, Methodology, Investigation, Resources, Formal analysis, Investigation, Resources, Writing - Review \& Editing. All authors read and approved the final manuscript.

\section{Funding}

This study was supported by Kafkas University Scientific Research Projects (2016-FM-24).

\section{Compliance with ethical standards}

\section{Conflict of interest}

Page 14/19 
The authors declare that they have no competing interests.

\section{Ethical approval}

This article does not contain any studies with human participants or animals performed by any of the authors.

\section{Methods}

Methods are incorporated into Supplementary Information

\section{Data availability}

The datasets generated during and/or analyzed during the current study are available from supplementary information files and the corresponding author on reasonable request.

\section{References}

1. Aanniz, T., Ouadghiri, M., Melloul, M., Swings, J., Elfahime, E., Ibijbijen, J., Ismaili, M. and Amar, M. (2015) 'Thermophilic bacteria in Moroccan hot springs, salt marshes and desert soils', Brazilian Journal of Microbiology, 46(2), 443-53.

2. Abdollahi, P., Ghane, M. and Babaeekhou, L. (2021) 'Isolation and Characterization of Thermophilic Bacteria from Gavmesh Goli Hot Spring in Sabalan Geothermal Field, Iran: Thermomonas hydrothermalis and Bacillus altitudinis Isolates as a Potential Source of Thermostable Protease', Geomicrobiology Journal, 38(1), 87-95.

3. Abootalebi, S. N., Saeed, A., Gholami, A., Mohkam, M., Kazemi, A., Nezafat, N., Mousavi, S. M., Hashemi, S. A. and Shorafa, E. (2020) 'Screening, characterization and production of thermostable alpha-amylase produced by a novel thermophilic Bacillus megaterium isolated from pediatric intensive care unit', Journal of Environmental Treatment Techniques, 8(3), 952-960.

4. Acer, Ö., Pırınççiğlu, H., Bekler, F. M. and Gül-Güven, R. (2015) 'Anoxybacillus sp. AH1, an a-amylase-producing thermophilic bacterium isolated from Dargeçit hot spring', Biologia, 70(7), 853-862.

5. Adiguzel, A., Inan, K., Sahin, F., Arasoglu, T., Gulluce, M., Belduz, A. O. and Baris, O. (2011) 'Molecular diversity of thermophilic bacteria isolated from Pasinler hot spring (Erzurum, Turkey)', Turkish Journal of Biology, 35(3), 267-274.

6. Akita, H., Kimura, Z.-i. and Matsushika, A. (2017) 'Complete Genome Sequence of Ureibacillus thermosphaericus A1, a Thermophilic Bacillus Isolated from Compost', Genome Announcements, 5(38), e00910-17.

7. Akmar, H. N., Asma, I., Venugopal, B., Latha, L. Y. and Sasidharan, S. (2011) 'Identification of appropriate sample and culture method for isolation of new thermophilic bacteria from hot spring', African Journal of Microbiology Research, 5(3), 217-221.

8. Al-Johani, N. B., Al-Seeni, M. N. and Ahmed, Y. M. (2016) 'Optımızatıon of alkaline a-amylase production by Thermophilic Bacillus subtilis', African journal of traditional, complementary, and alternative medicines : AJTCAM, 14(1), 288-301.

9. Baltaci, M. O., Genc, B., Arslan, S., Adiguzel, G. and Adiguzel, A. (2017) 'Isolation and Characterization of Thermophilic Bacteria from Geothermal Areas in Turkey and Preliminary Research on Biotechnologically Important Enzyme Production', Geomicrobiology Journal, 34(1), 53-62.

10. Bernard, K., Burdz, T., Wiebe, D., Balcewich, B. M., Zimmerman, T., Lagacé-Wiens, P., Hoang, L. M. and Bernier, A.-M. J. A. (2017) 'Characterization of isolates of Eisenbergiella tayi, a strictly anaerobic Gram-stain variable bacillus recovered from human clinical materials in Canada', 44, 128-132.

11. Bernardo, S. P. C., Rosana, A. R. R., de Souza, A. N., Chiorean, S., Martins, M. L. L. and Vederas, J. C. (2020) 'Draft Genome Sequence of the Thermophilic Bacterium Bacillus licheniformis SMIA-2, an Antimicrobial- and Thermostable Enzyme-Producing Isolate from Brazilian Soil', Microbiology Resource Announcements, 9(17), e00106-20.

12. Bouacem, K., Amziane-Touazi, M., Hania, W. B., Cayol, J., Fardeau, M., Benayad, T., Hacene, H. and Bouanane-Darenfed, A. (2021) 'Isolation and characterization of moderately thermophilic aerobic cultivable bacteria from Hammam Righa Hot Spring (Algeria): Description of their hydrolytic capacities', Algerian Journal of Environmental Science and Technology.

13. Chakraborty, R. (2002) 'Molecular evolution and phylogenetics, Masatoshi Nei and Sudhir Kumar. Oxford University Press, Oxford, England/New York, USA, 2000, xiv+333 pages (hardback) \$75; (paperback) \$35.00', Molecular Phylogenetics and Evolution, 25(3), 569-570.

14. Chan, C. S., Chan, K. G., Tay, Y. L., Chua, Y. H. and Goh, K. M. (2015) 'Diversity of thermophiles in a Malaysian hot spring determined using 16S rRNA and shotgun metagenome sequencing', Front Microbiol, 6, 177.

15. Dang, T. C. H., Nguyen, D. T., Thai, H., Nguyen, T. C., Tran, T. T. H., Le, V. H., Van Huynh, N., Tran, X. B., Pham, T. P. T., Nguyen, T. G. and Nguyen, Q. T. (2018) 'Plastic degradation by thermophilic Bacillus sp. BCBT21 isolated from composting agricultural residual in Vietnam', Advances in Natural Sciences Nanoscience and Nanotechnology, 9(1), 015014.

16. Derya, Y. and Ahmet, A. (2014) 'Molecular Typing of Thermophilic Bacilli isolated from Different Hot Springs of Turkey', Research Journal of Biotechnology, 9(10), 83-88.

17. Eriksen, N. T. (2008) 'Production of phycocyanin-a pigment with applications in biology, biotechnology, foods and medicine', App/ Microbiol Biotechnol, $80(1), 1-14$. 
18. Ertuğrul, S., Dönmez, G. and Takaç, S. (2007) 'Isolation of lipase producing Bacillus sp. from olive mill wastewater and improving its enzyme activity', $J$ Hazard Mater, 149(3), 720-4.

19. Falcicchio, P., Levisson, M., Kengen, S. W., Koutsopoulos, S. and van der Oost, J. (2021) '(Hyper) thermophilic enzymes: Production and purification' in Protein Downstream Processing, Springer, 469-478.

20. Felsenstein, J. (1985) 'CONFIDENCE LIMITS ON PHYLOGENIES: AN APPROACH USING THE BOOTSTRAP', Evolution, 39(4), $783-791$.

21. Gormez, A., Sahin, F., Gulluce, M. and Aslan, I. (2013) 'Identification and characterization of Pseudomonas syringae isolated from apricot trees in the erzurum province of Turkey and evaluation of cultivar reaction', Journal of Plant Pathology, 525-532.

22. Guven, K., Matpan Bekler, F. and Gul Guven, R. (2018) 'Thermophilic and Halophilic Microorganisms Isolated from Extreme Environments of Turkey, with Potential Biotechnological Applications' in Egamberdieva, D., Birkeland, N.-K., Panosyan, H. and Li, W.-J., eds., Extremophiles in Eurasian Ecosystems: Ecology, Diversity, and Applications, Singapore: Springer Singapore, 219-264.

23. https://blast.ncbi.nlm.nih.gov/Blast.cgi 2020.

24. https://www.ezbiocloud.net/ 2020.

25. Ifandi, S. and Alwi, M. (2018) 'Isolation of thermophilic bacteria from Bora hot springs in Central Sulawesi', Journal of Biology Biology Education, 10(2), 291-297.

26. Jacobus, A. P. and Gross, J. (2015) 'Optimal cloning of PCR fragments by homologous recombination in Escherichia coli', PLoS One, 10 (3), e0119221.

27. Khan, M. and Sathya, T. A. (2018) 'Extremozymes from metagenome: Potential applications in food processing', Crit Rev Food Sci Nutr, 58(12), $2017-2025$.

28. Kılınç, E., Ozdemir, S., Yalçın, M. S. and Soylak, M. (2021) 'A new method for the preconcentrations of U(VI) and Th(IV) by magnetized thermophilic bacteria as a novel biosorbent', Analytical and Bioanalytical Chemistry, 413(4), 1107-1116.

29. Kumar, S., Stecher, G. and Tamura, K. (2016) 'MEGA7: Molecular Evolutionary Genetics Analysis Version 7.0 for Bigger Datasets', Molecular biology and evolution, 33(7), 1870-4.

30. Ladeira, S. A., Cruz, E., Delatorre, A. B., Barbosa, J. B. and Martins, M. L. L. (2015) 'Cellulase production by thermophilic Bacillus sp. SMIA-2 and its detergent compatibility', Electronic Journal of Biotechnology, 18(2), 110-115.

31. Lee, H. S., Kwon, K. K., Kang, S. G., Cha, S.-S., Kim, S.-J. and Lee, J.-H. (2010) 'Approaches for novel enzyme discovery from marine environments', Current Opinion in Biotechnology, 21(3), 353-357.

32. Li, X., Zhao, X., Tian, D., Zhu, Y. and Yao, T. (1999) 'A new method for PCR products cloning-T-A clone technique', Sheng wu hua xue yu sheng wu wu li jin zhan, 26(2), 187-189.

33. Luo, Z., Miao, J., Li, G., Du, Y. and Yu, X. (2017) 'A Recombinant Highly Thermostable $\beta$-Mannanase (ReTMan26) from Thermophilic Bacillus subtilis (TBS2) Expressed in Pichia pastoris and Its pH and Temperature Stability', Applied Biochemistry and Biotechnology, 182(4), $1259-1275$.

34. Margaryan, A., Shahinyan, G., Hovhannisyan, P., Panosyan, H., Birkeland, N.-K. and Trchounian, A. (2018) 'Geobacillus and Anoxybacillus spp. from Terrestrial Geothermal Springs Worldwide: Diversity and Biotechnological Applications' in Egamberdieva, D., Birkeland, N.-K., Panosyan, H. and Li, W.-J., eds., Extremophiles in Eurasian Ecosystems: Ecology, Diversity, and Applications, Singapore: Springer Singapore, 119-166.

35. Mehta, R., Singhal, P., Singh, H., Damle, D. and Sharma, A. K. (2016) 'Insight into thermophiles and their wide-spectrum applications', 3 Biotech, 6(1), 81-81.

36. Mohammad, B. T., Al Daghistani, H. I., Jaouani, A., Abdel-Latif, S. and Kennes, C. (2017) 'Isolation and Characterization of Thermophilic Bacteria from Jordanian Hot Springs: Bacillus licheniformis and Thermomonas hydrothermalis Isolates as Potential Producers of Thermostable Enzymes', International Journal of Microbiology, 2017, 6943952.

37. Msarah, M. J., Ibrahim, I., Hamid, A. A. and Aqma, W. S. (2020) 'Optimisation and production of alpha amylase from thermophilic Bacillus spp. and its application in food waste biodegradation', Heliyon, 6(6), e04183.

38. Mutlu, H. and Güleç, N. (1998) 'Hydrogeochemical outline of thermal waters and geothermometry applications in Anatolia (Turkey)', Journal of Volcanology and Geothermal Research, 85(1), 495-515.

39. Oztas Gulmus, E. and Gormez, A. (2020a) 'Characterization and biotechnological application of protease from thermophilic Thermomonas haemolytica', Archives of Microbiology, 202(1), 153-159.

40. Oztas Gulmus, E. and Gormez, A. (2020b) 'Identification and Characterization of Novel Thermophilic Bacteria from Hot Springs, Erzurum, Turkey', Current Microbiology, 77(6), 979-987.

41. Panosyan, H., Margaryan, A. and Birkeland, N.-K. (2020) 'Geothermal springs in Armenia and Nagorno-Karabakh: potential sources of hydrolase-producing thermophilic bacilli', Extremophiles, 24 (4), 519-536.

42. Poddar, A. and Das, S. K. (2018) 'Microbiological studies of hot springs in India: a review', Archives of Microbiology, 200(1), 1-18.

43. Poli, A., Guven, K., Romano, I., Pirinccioglu, H., Guven, R. G., Euzeby, J. P., Matpan, F., Acer, O., Orlando, P. and Nicolaus, B. (2012) 'Geobacillus subterraneus subsp. aromaticivorans subsp. nov., a novel thermophilic and alkaliphilic bacterium isolated from a hot spring in Sirnak, Turkey', J Gen Appl Microbiol, $58(6), 437-46$.

44. Prescott, H. (2002) 'Laboratory Exercises in Microbiology Fifth Edition',

45. Prescott, L. M. and Harley, J. P. (2002) 'Harley Prescott: Laboratory Exercises in Microbiology, Fifth Editio',

46. Saeed, A. M., Sayed, H. A. E. and El-Shatoury, E. H. (2020) 'Optimizing the Reduction of Molybdate by Two Novel Thermophilic Bacilli Isolated from Sinai, Egypt', Current Microbiology, 77(5), 786-794.

47. Safitri, R., Kusumawardhani, D. P., Annisa, A., Partasasmita, R., Asharina, S. and Maskoen, A. M. (2020) 'Characterization and identification of three thermophilic Bacillus strain isolated from Domas Crater, Mt. Tangkuban Perahu, Indonesia', Biodiversitas Journal of Biological Diversity, 21(8).

Page 16/19 
48. Saitou, N. and Nei, M. (1987) 'The neighbor-joining method: a new method for reconstructing phylogenetic trees', Molecular biology and evolution, 4(4), 406-425.

49. Saleh, F., Hussain, A., Younis, T., Ali, S., Rashid, M., Ali, A., Mustafa, G., Jabeen, F., Al-Surhanee, A. A., Alnoman, M. M. and Qamer, S. (2020) 'Comparative growth potential of thermophilic amylolytic Bacillus sp. on unconventional media food wastes and its industrial application', Saudi Journal of Biological Sciences, 27(12), 3499-3504.

50. Salem, R. B., Abbassi, M. S., Cayol, J.-I., Bourouis, A., Mahrouki, S., Fardeau, M.-L. and Belhadj, O. (2020) 'Thermophilic Bacillus licheniformis RBS 5 isolated from hot Tunisian spring co-producing alkaline and thermostable a-amylase and protease enzymes', Journal of Microbiology, Biotechnology and Food Sciences, 5(6), 557-562.

51. Sambrook, J. J. M. c. (1987) 'Commonly used techniques in molecular cloning', 3, E1-E39.

52. Savas, S., Adiguzel, A., Inan, K., Ozkan, H., Gulluce, M. and Sahin, F. (2009) 'Molecular characterization of thermophilic bacteria isolated from Van City Ercis Town Hasanabdal hot spring', Romanian Biotechnological Letters, 14(3), 4445-4454.

53. Shen, P., Niu, D., Liu, X., Tian, K., Kugenthiren, P., Singh, S. and Wang, Z. (2021) 'Overexpression of Thermophilic a-amylase in Bacillus Licheniformis using a High Efficiency Chromosomal Integration and Amplification Strategy', Microbial Cell Factories.

54. Short, J. M. (1998) 'Directed evolution of thermophilic enzymes',

55. Tango, M. S. A. and Islam, M. R. (2002) 'Potential of Extremophiles for Biotechnological and Petroleum Applications', Energy Sources, $24(6), 543-559$.

56. Thakur, A., Sharma, K., Mutreja, R. and Goyal, A. (2021) 'Thermostable Enzymes from Clostridium thermocellum' in Bioprospecting of Enzymes in Industry, Healthcare and Sustainable Environment, Springer, 251-267.

57. Tirawongsaroj, P., Sriprang, R., Harnpicharnchai, P., Thongaram, T., Champreda, V., Tanapongpipat, S., Pootanakit, K. and Eurwilaichitr, L. (2008) 'Novel thermophilic and thermostable lipolytic enzymes from a Thailand hot spring metagenomic library', Journal of Biotechnology, 133(1), 42-9.

58. Verma, J. P., Jaiswal, D. K., Krishna, R., Prakash, S., Yadav, J. and Singh, V. (2018) 'Characterization and Screening of Thermophilic Bacillus Strains for Developing Plant Growth Promoting Consortium From Hot Spring of Leh and Ladakh Region of India', Front Microbiol, 9, 1293.

59. Wang, L., Tang, Y., Wang, S., Liu, R. L., Liu, M. Z., Zhang, Y., Liang, F. L. and Feng, L. (2006) 'Isolation and characterization of a novel thermophilic Bacillus strain degrading long-chain n-alkanes', Extremophiles, 10(4), 347-56.

60. Yadav, P., Korpole, S., Prasad, G. S., Sahni, G., Maharjan, J., Sreerama, L. and Bhattarai, T. (2018) 'Morphological, enzymatic screening, and phylogenetic analysis of thermophilic bacilli isolated from five hot springs of Myagdi, Nepal', J. Appl. Biol. Biotechnol, 6(3), 1-8.

61. Yanmis, D., Baltaci, M. O., Gulluce, M. and Adiguzel, A. (2015) 'Identification of thermophilic strains from geothermal areas in Turkey by using conventional and molecular techniques', Research Journal of Biotechnology, 10(1), 39-45.

62. Yilmaz, B., Baltaci, M. O., Sisecioglu, M. and Adiguzel, A. (2016) 'Thermotolerant alkaline protease enzyme from Bacillus licheniformis A10: purification, characterization, effects of surfactants and organic solvents', Journal of Enzyme Inhibition and Medicinal Chemistry, 31(6), $1241-1247$.

63. Zakzeski, J., Bruijnincx, P. C., Jongerius, A. L. and Weckhuysen, B. M. (2010) 'The catalytic valorization of lignin for the production of renewable chemicals', Chem Rev, 110(6), 3552-99.

\section{Figures}

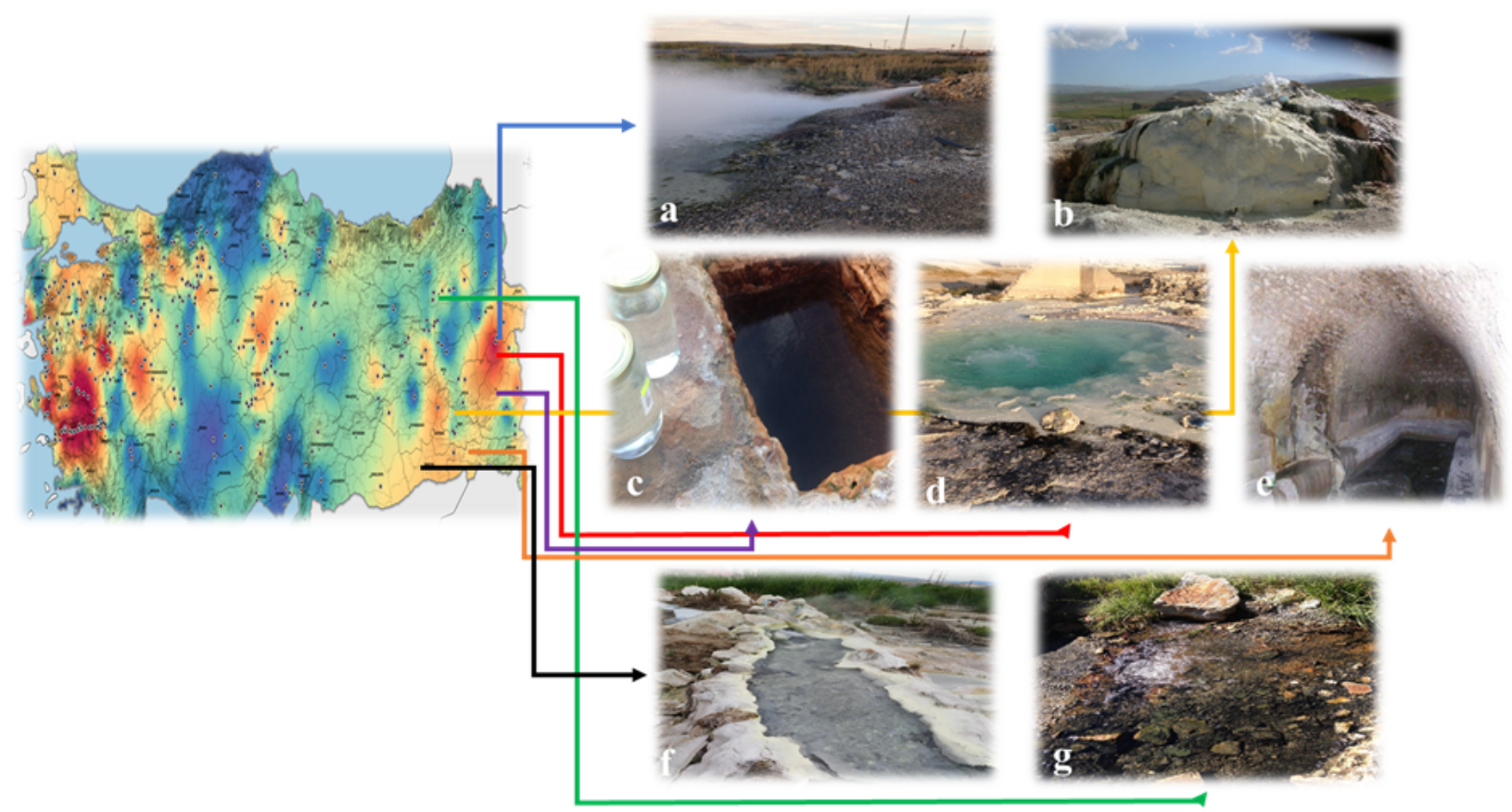

Figure 1

Page 17/19 
Referring to the Turkey map, hot springs under study are indicated by arrows. Source of geothermal hot springs: a. Kopru (Agri), see Supplemantal movie 1., b. Hista (Siirt), c. Hasanabdal (Van), d. Davut (Agri), e. Guclukonak (Sirnak), f. Dargecit (Mardin), g. Pasinler (Erzurum) (https://www.mta.gov.tr). Note: The designations employed and the presentation of the material on this map do not imply the expression of any opinion whatsoever on the part of Research Square concerning the legal status of any country, territory, city or area or of its authorities, or concerning the delimitation of its frontiers or boundaries. This map has been provided by the authors.

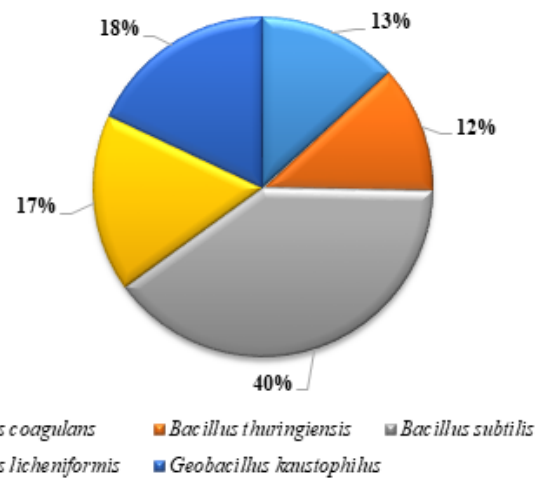

\section{Figure 2}

Pie chart showing the distribution of bacteria resulting from sequencing in hot springs

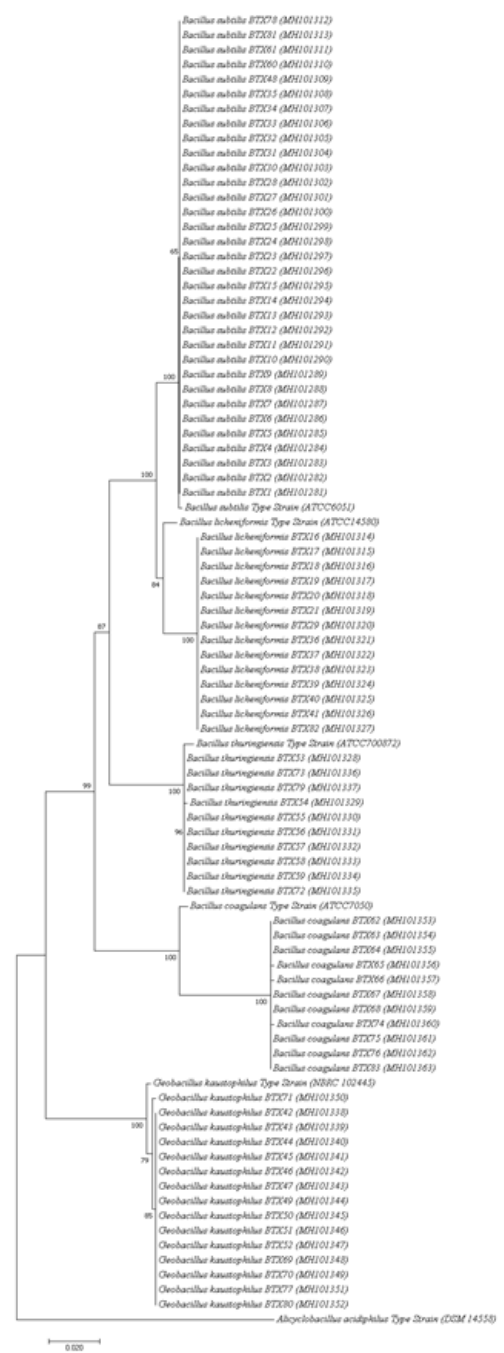

Figure 3

The evolutionary relationship was inferred using the Neighbor-Joining method [58]. The optimal tree with the sum of branch length $=0.34395833$ was shown. The percentage of replicate trees in which the associated taxa clustered together in the bootstrap test (1000 replicates) is shown next to the branches [59]. The 
analysis of 89 nucleotide sequences (83 isolates, 5 type strains, and 1 outgroup) was conducted in MEGA7 [61]. As shown in the figure, bacteria belonging to the genus Bacillus were divided into different groups from the genus Geobacillus, to be a single group with type strains as expected. =Cellulase $\|$ Lipase $\|$ Protease $\|$ Amylase
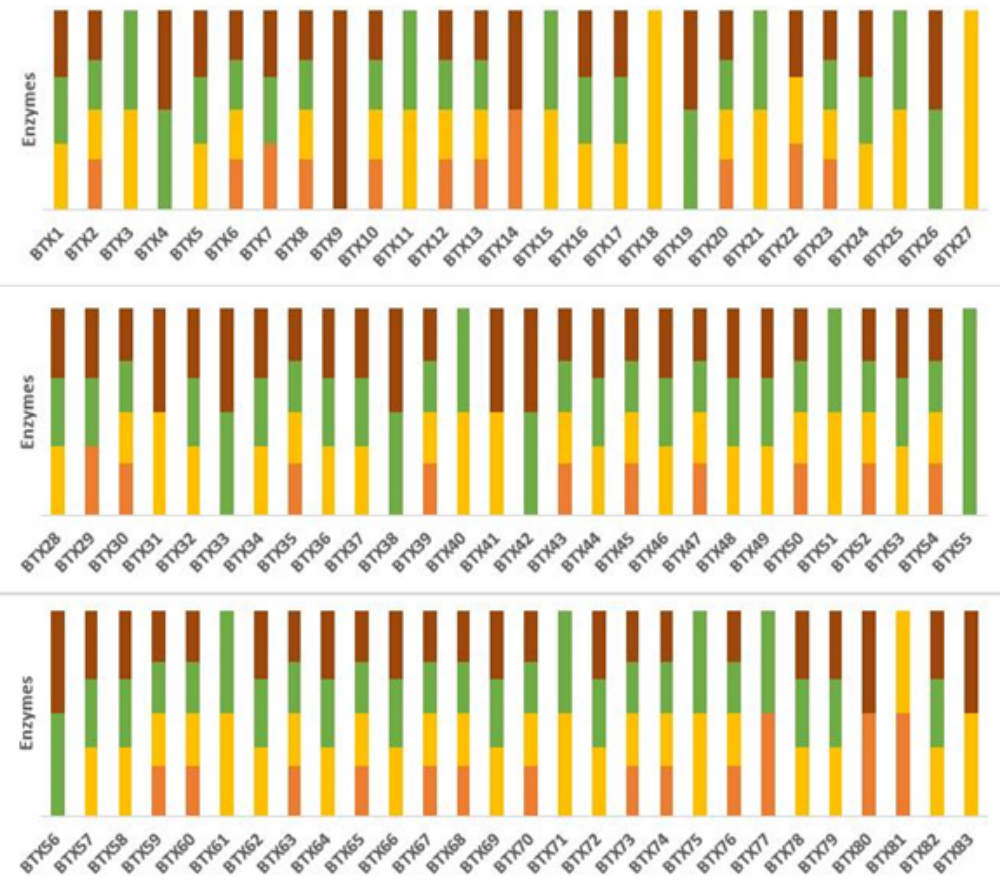

Figure 4

According to the qualitative analysis results, it was determined that $27(32.8 \%)$ isolates can produce amylase, protease, cellulase, and lipase. In the study, 68 (82\%), 73 (88\%), 34 (41\%), and 69 (83\%) isolates were determined as producers of amylase, protease, cellulase, and lipase, respectively.

\section{Supplementary Files}

This is a list of supplementary files associated with this preprint. Click to download.

- Movie1.docx 